OPEN

SUBJECT AREAS:

COMPUTATIONAL

BIOLOGY AND

BIOINFORMATICS

GENE REGULATORY

NETWORKS

Received

23 April 2014

Accepted

13 May 2014

Published

5 June 2014

Correspondence and requests for materials should be addressed to

K.E.K. (karl.kadler@ manchester.ac.uk) or

Q.J.M. (qing-jun. meng@manchester.ac.

uk)

\section{Gremlin-2 is a BMP antagonist that is regulated by the circadian clock}

\author{
Ching-Yan Chloé Yeung ${ }^{1,2}$, Nicole Gossan 1,2, Yinhui Lu 1,2, Alun Hughes², James J. Hensman ${ }^{3}$, \\ Monika L. Bayer ${ }^{4}$, Michael Kiær ${ }^{4}$, Karl E. Kadler ${ }^{1,2}$ \& Qing-Jun Meng ${ }^{1,2}$
}

\begin{abstract}
'Wellcome Trust Centre for Cell-Matrix Research, University of Manchester, Oxford Road, Manchester M13 9PT, United Kingdom, ${ }^{2}$ Faculty of Life Sciences, University of Manchester, Oxford Road, Manchester M13 9PT, United Kingdom, ${ }^{3}$ Department of Computer Science, University of Sheffield, Sheffield S1 4DP, United Kingdom, ${ }^{4}$ Institute of Sports Medicine, Department of Orthopedic Surgery M, Bispebjerg Hospital, and Center for Healthy Aging, Faculty of Health Sciences, University of Copenhagen, 2400 Copenhagen, Denmark.
\end{abstract}

Tendons are prominent members of the family of fibrous connective tissues (FCTs), which collectively are the most abundant tissues in vertebrates and have crucial roles in transmitting mechanical force and linking organs. Tendon diseases are among the most common arthropathy disorders; thus knowledge of tendon gene regulation is essential for a complete understanding of FCT biology. Here we show autonomous circadian rhythms in mouse tendon and primary human tenocytes, controlled by an intrinsic molecular circadian clock. Time-series microarrays identified the first circadian transcriptome of murine tendon, revealing that $4.6 \%$ of the transcripts ( 745 genes) are expressed in a circadian manner. One of these genes was Grem2, which oscillated in antiphase to BMP signaling. Moreover, recombinant human Gremlin-2 blocked BMP2-induced phosphorylation of Smad1/5 and osteogenic differentiation of human tenocytes in vitro. We observed dampened Grem 2 expression, deregulated BMP signaling, and spontaneously calcifying tendons in young CLOCKA19 arrhythmic mice and aged wild-type mice. Thus, disruption of circadian control, through mutations or aging, of Grem2/BMP signaling becomes a new focus for the study of calcific tendinopathy, which affects 1 -in-5 people over the age of 50 years.

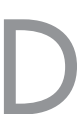

iseases and injury to tendons are a major biomedical problem. Rotator cuff disease, repetitive motion injuries, complications following iatrogenic injury, and tendon rupture, collectively affect 1-in-3 people over the age of 40 years and incur a significant sociological and economic burden (see ${ }^{1,2}$ and references therein). Calcium deposits in the mid-substance of the tendon are common in rotator cuff tendons, Supraspinatus, Achilles and patellar tendons resulting in calcific tendinopathy (CT), which has a prevalence of between $2.7 \%$ and $22 \%$ depending on age and gender (reviewed by ${ }^{3-7}$ ). Thus, tendinopathies are the most common of the arthropathy disorders. However, the pathophysiology is not always associated with overuse, indicative of an underlying defect in tissue homeostasis as a result of age. An under-researched aspect is the dramatic differences in physiological activity of tendons during a 24-hour cycle of being asleep and awake, and if these differences are influenced by aging and gene expression changes.

The circadian clock coordinates the 24-hourly rhythms in biochemical and behavioral parameters that help prepare an organism for anticipated changes in its environment. In addition to the central pacemaker in the suprachiasmatic nuclei (SCN), many peripheral organs contain autonomous peripheral clocks ${ }^{8,9}$. Mammalian circadian clocks rely on the transcriptional/translational feedback loops consisting of the transcriptional activator complex CLOCK and BMAL1 (bone and muscle ARNT-like 1), and repressor complex PERIOD1/2 and CRY1/2. PERs/CRYs periodically inhibit the CLOCK/BMAL1-mediated transactivation of the Pers and Crys through Ebox elements in their promoters. The core oscillator then drives rhythmic clock-controlled genes through regulatory elements in their promoters (e.g. E-box, D-box, and ROREs).

Importantly, mice with a deletion of Bmall show age-related and non-inflammatory arthropathy, including ectopic tendon calcification ${ }^{10}$. It was unknown if the tendon phenotype was the result of Bmall gene-specific effects or to disruptions to the molecular clock, and if the latter, if tendon contains a functional clock that controls tissue homeostasis. Here, we show for the first time the presence of self-sustained circadian clocks in mouse tendon and human tenocytes, and, the circadian clock controls BMP signaling. The results indicate that tendon clocks control 4.6\% of local transcripts including Grem2, which we show is an antagonist of BMP signaling and inhibits calcification of primary human tenocytes in culture. Disruption of the circadian rhythms in the arrhythmic CLOCK 19 mice $^{11}$ is associated with aberrant Grem2 expression and BMP signaling, calcific pathology and 

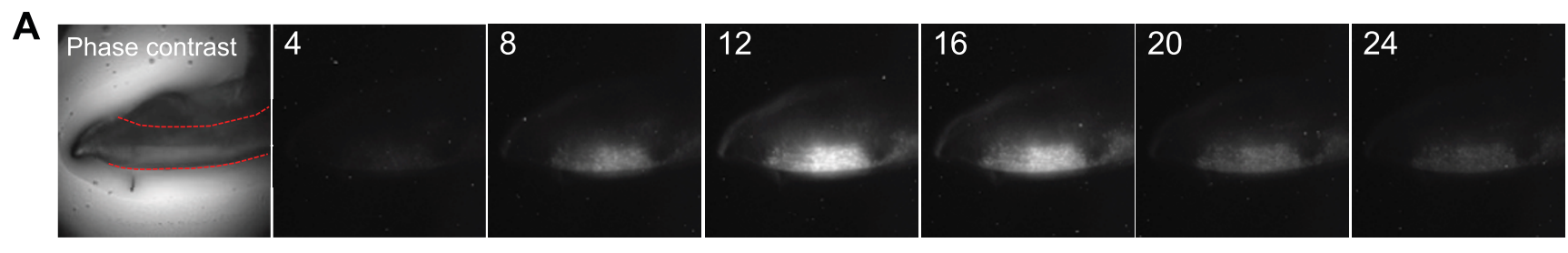

B

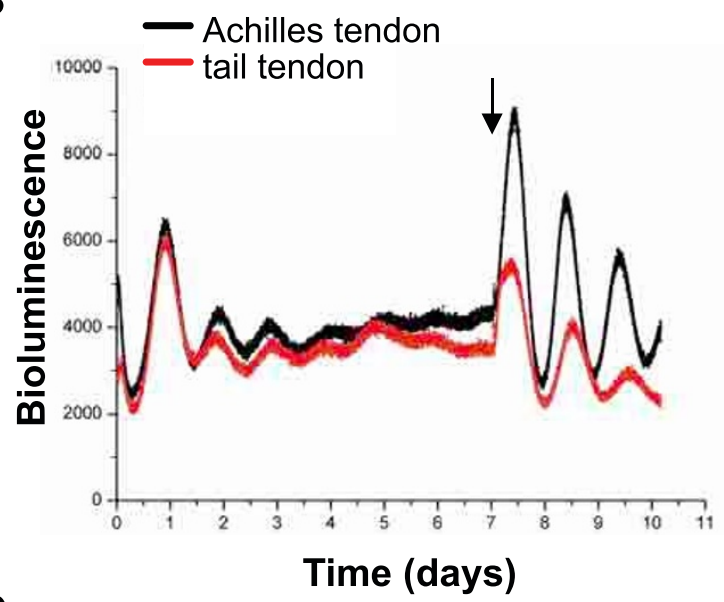

D

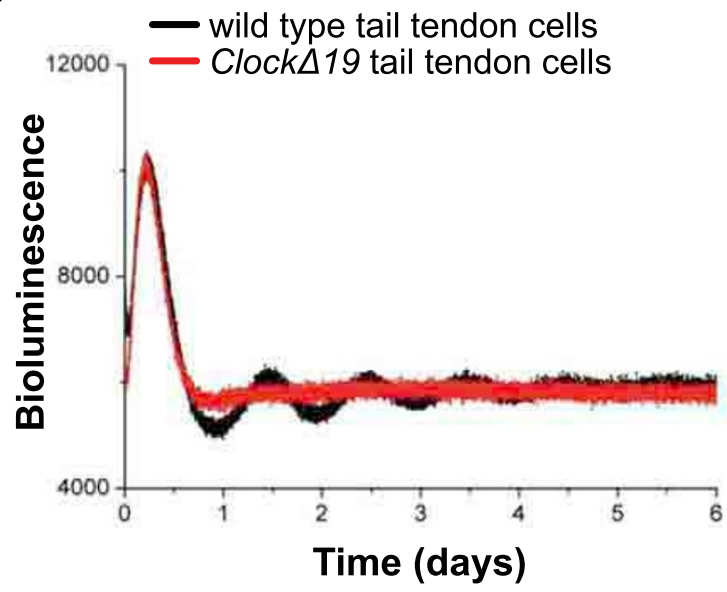

C

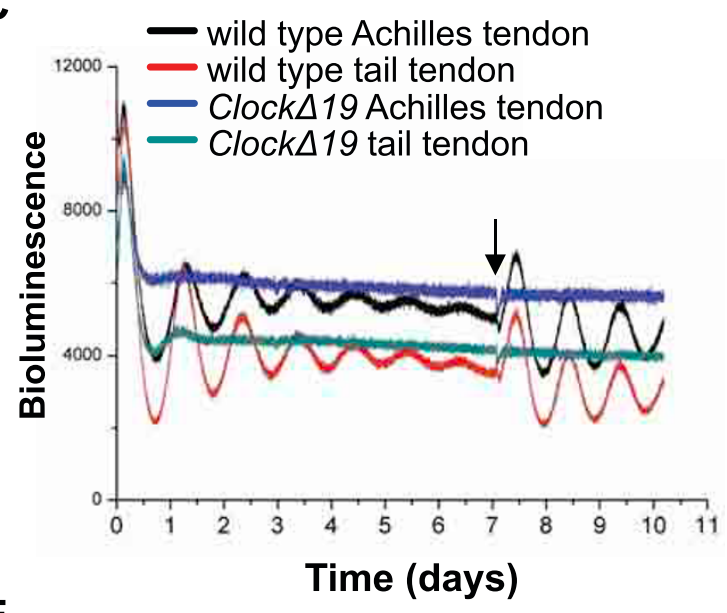

$\mathbf{E}$

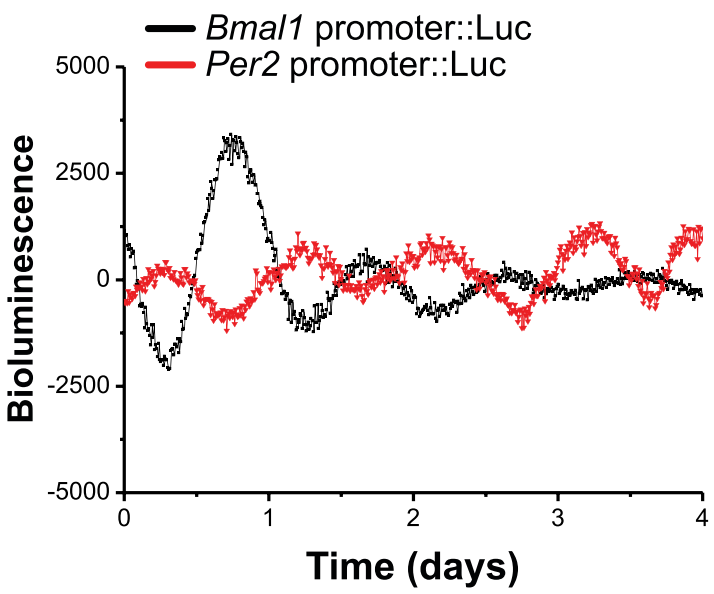

Figure 1 Tendon tissues and cells have an autonomous circadian rhythm. (A) Bioluminescence microscopy of dissected Achilles tendon from PER2::Luc reporter mouse imaged in the presence of $100 \mathrm{nM}$ dexamethasone. Image of the dissected Achilles tendon under phase contrast microscopy is shown, where dotted red lines outline the tendon shaft. (B-D) PMT recordings of endogenous circadian rhythms and re-initiation of the rhythms with $100 \mathrm{nM}$ dexamethasone (arrow) in dissected Achilles and tail tendons from PER2::Luc mice (B); compared with tendons from CLOCK $\Delta 19$ mice bred on a PER2::Luc background (C); and in cultured tail tendon cells isolated from wild type and CLOCK $\Delta 19$ x PER2::Luc mice (D). (E) PMT recordings of Per2::Luc and Bmal1::Luc reporters expressed in primary human tendon cells. Representative readings from one preparation of cells are shown. Arrow indicates re-initiation of rhythm with $100 \mathrm{nM}$ dexamethasone.

mechanically weak tendons. Finally, we show that aged wild-type mice exhibit a dampened and delayed tendon circadian rhythm associated with profound calcification.

\section{Results}

Autonomous circadian rhythm in tendon. To investigate if tendon has an intrinsic clock we examined Achilles and tail tendons from PER2::Luc reporter mice ${ }^{12}$ and used real-time bioluminescence microscopy and a photomultiplier tube (PMT) to record light emission. This revealed robust circadian rhythms of PER2::Luc activity, with periods of $23.73 \pm 0.26 \mathrm{hr}$ and $23.23 \pm 0.06 \mathrm{hr}$ in Achilles and tail tendons, respectively, indicating that there is a circadian clock in Achilles and tail tendon (Figure 1A and B, and Supplementary video). As expected, the rhythm dampened over time in culture but was effectively reinstated after a single treatment with dexamethasone, a known synchronizing agent for peripheral clocks.

We next examined the circadian rhythm of Achilles and tail tendons from the CLOCK $\Delta 19$ x PER2::Luc crosses. The CLOCK $\Delta 19$ mice harbor a deletion in exon 19 of the CLOCK gene producing a dominant negative mutant protein ${ }^{11}$. The results confirmed that CLOCK $\Delta 19$ tendons are arrhythmic (Figure 1C). We also established that tendon cells isolated from wild type but not CLOCK $\Delta 19$ tail tendons have an autonomous circadian rhythm (Figure 1D). To extend these studies to human tendon cells, primary human tenocytes were transduced with lentivirus carrying Bmal1- or Per2-promoter-driven luciferase reporters. Both reporters showed circadian oscillation in anti-phase to one another (Figure 1E). 


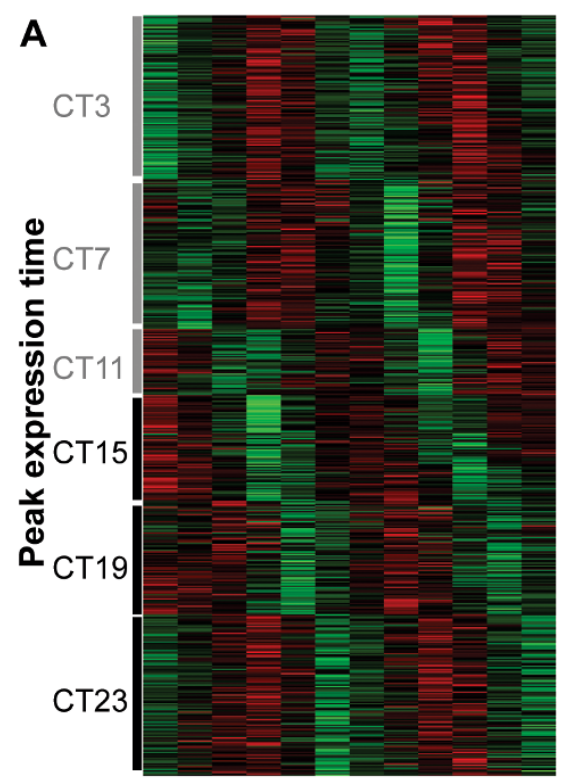

Hrs in DD: 394347515559636771757983

Key: $\min \square \max$

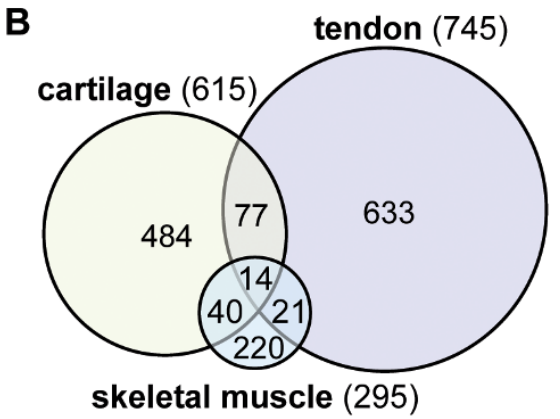

C

$\ldots$ tail tendon microarray data

- - tail tendon qPCR

- Achilles tendon qPCR
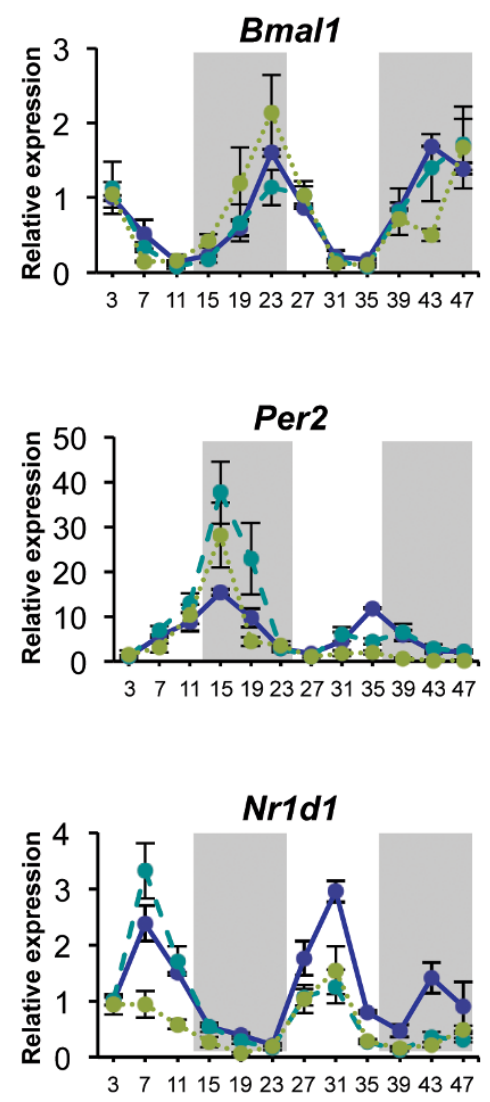

D

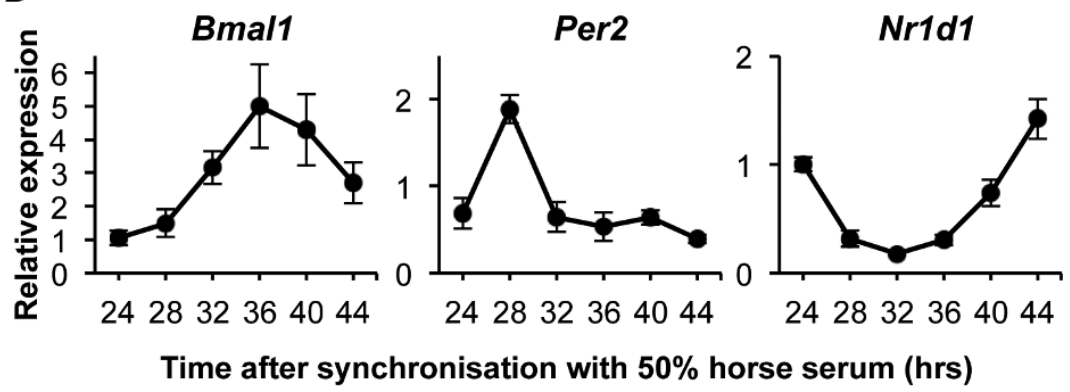

Figure $2 \mid$ Circadian transcriptome in tendon. (A) Heat map depicting the expression level of the 745 circadian genes (4.6\% of the tendon transcriptome) identified by Circwave Batch and JTKCycle. Genes are organized according to timing of peak expression in circadian time $(\mathrm{CT})$. DD $=$ hours in dark/dark cycle. Grey bars represent subjective day; black bars represent subjective night. (B) Specificity of tendon clock genes. Venn diagram comparing the number of circadian genes of tendon, cartilage ${ }^{9}$ and skeletal muscle ${ }^{8}$. The total number of genes identified as circadian in each tissue is represented in brackets; areas of overlap indicate common genes. (C) qPCR validation of time-dependent expression of clock genes, Bmal1, Per2, and Nr1d1 (REV$E R B \alpha)$ in mouse tendons. $q P C R$ values $(\mathrm{n}=6$ for tail tendons; $\mathrm{n}=4$ for Achilles tendons) normalized to GAPDH. Microarray data of tail tendon $(\mathrm{n}=2)$ was plotted for comparison. Grey shadow indicates subjective night phase. (D) Time-dependent expression of clock genes, Bmall, Per2 and Nr1d1 (REV$E R B \alpha)$ in primary human tendon cells $(\mathrm{n}=3)$. Cells were synchronized with $50 \%$ horse serum and gene expression was analyzed by $\mathrm{qPCR}$. $\mathrm{qPCR}$ values normalized to GAPDH. Mean data from three preparations of cells shown. Error bars indicate SEM.

The circadian transcriptome in tendon. Circadian transcriptome studies in muscle, liver and cartilage have demonstrated very low numbers of overlapping rhythmic genes, highlighting the tissuespecific functions of the peripheral circadian clocks (for discussion $\mathrm{see}^{9}$ ). To gain further insights into the functions of the tendon clock, we performed a time-series microarray to identify the circadianlyregulated genes. Female C57BL/6 mice were kept in constant dark- ness for $39 \mathrm{hr}$ to reveal genes driven by the endogenous circadian clock rather than by light-related cues. Tail tendon was taken at 4-hr intervals during $48 \mathrm{hr}$. In total, 16,000 genes (represented by 29,000 probes) were classified as expressed. To identify circadian transcripts, we used two well-recognized algorithms based on different statistical models as described previously ${ }^{9}$. CircWave Batch revealed $\sim 1000$ probes oscillating with a period of $\sim 24 \mathrm{hr}$ (Figure $2 \mathrm{~A}$ and 
A

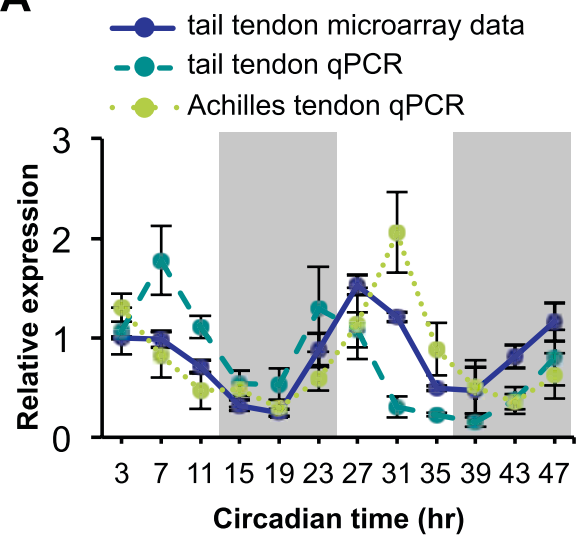

C

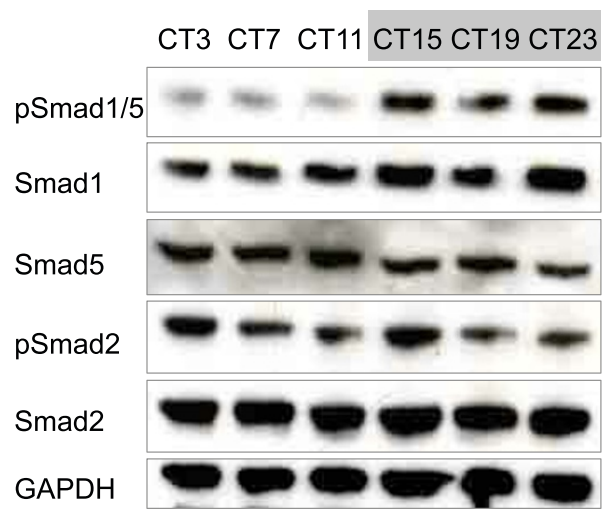

B

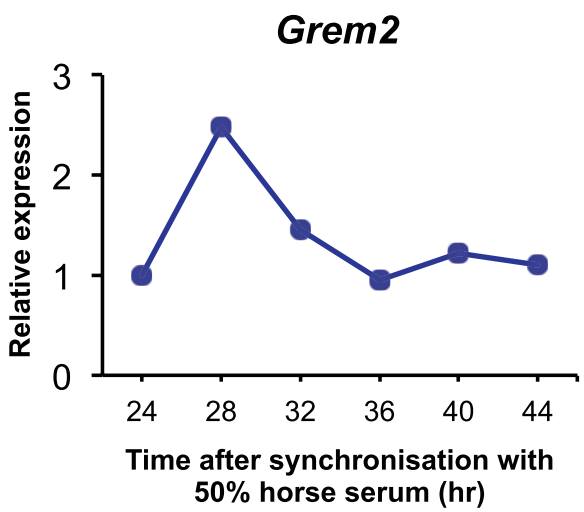

D

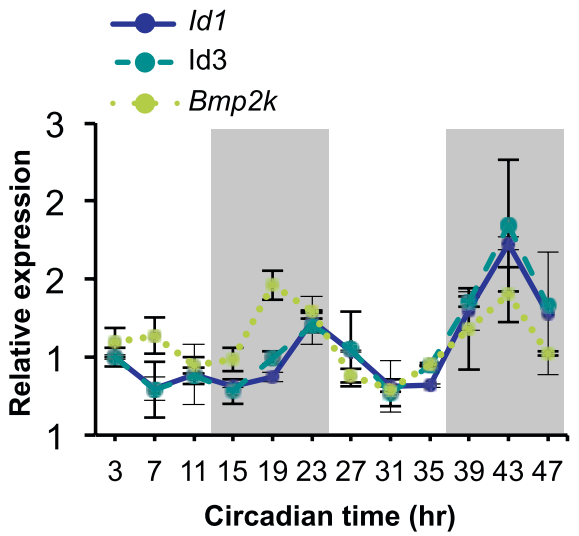

Figure 3 Circadian control of Grem2 expression and BMP signaling in mouse tendon. (A) Time-dependent expression of Grem2 from the microarray analysis of tail tendon $(n=2)$ and qPCR validations in tail $(n=6)$ and Achilles tendons $(n=4)$. (B) Time-dependent expression of Grem2 in primary human tendon cells. Representative data from one preparation of cells shown. Cells were synchronized with $50 \%$ horse serum and gene expression was analyzed by qPCR. (C) Wild type mice were kept in DD for $36 \mathrm{hr}$ to allow endogenous circadian rhythm of gene expression. Protein was purified from tail tendons at 4-hr intervals and phosphorylation of Smad1/5 were analyzed by western blotting. Levels of GAPDH protein served as a loading control. Full-length western blots are shown in Supplementary Figure S4. (D) Endogenous expression pattern of BMP signaling target genes, Id 1, Id 3 and Bmp2k, in tail tendon over $44 \mathrm{hr}$ detected by time-series microarray $(\mathrm{n}=2)$. Error bars indicate SEM. Grey shadow indicates subjective night phase.

Figure S1, q $<0.003)$. The JTKcycle algorithm identified 1,500 rhythmic probes with $20-28 \mathrm{hr}$ period $(\mathrm{q}<0.1)$. To be stringent, we considered only those genes identified by both methods to be circadianly regulated. From these analyses we identified 745 genes (4.6\% of genes expressed in tendon) with rhythmic expression and a period of $\sim 24 \mathrm{hr}$ (Figure 2A). The uniqueness of the tendon circadian transcriptome is shown schematically in Figure $2 \mathrm{~B}$ in that only 14 genes are common between previously published circadianregulated genes in cartilage and skeletal muscle ${ }^{8,9}$. Among the 14 common genes were the core clock components Bmal1, Per2 and $N r 1 d 1$, whose circadian expression rhythms were validated by qPCR in mouse Achilles and tail tendon (Figure 2C). Using a Gaussian process model with a Bayesian approach ${ }^{9}$, rhythmic tendon genes were clustered into 9 distinct clusters according to their inferred periodic functions (Figure S2, Supplementary Table 2). Most genes peaked during late night and early day (Figure S3). In addition, expression of endogenous core clock genes, Bmal1, Per2 and Nr1d1 were rhythmically expressed in primary human tenocytes (Figure 2D). Thus, mouse and human tendon cells exhibit a cell autonomous circadian molecular oscillator.

Grem 2 as a clock controlled gene in tendon. One of the rhythmic genes in tendon was Grem2, with expression peaks in the beginning of day and is down-regulated at night (Figure $3 \mathrm{~A}$ and $\mathrm{B}$ ). The
Xenopus Gremlin is a secreted protein that can block BMP signaling by binding BMPs, thereby preventing receptor activation and phosphorylation of Smad $1 / 5^{13}$ (reviewed by ${ }^{14}$ and discussed in more detail below). Therefore, we predicted that the mammalian Grem2 might have a similar role in antagonizing BMP signaling. We used western blot analysis to assess levels of phosphorylated Smads in temporally-collected tail-tendon proteins (Figure 3C). Phosphorylation of Smad1/5 was observed at all 6 time points across one circadian cycle but was noticeably less abundant at CT3, CT7 and CT11 and more abundant at CT15, CT19 and CT23. The total amount of Smad1, Smad5, Smad2 and phosphorylated Smad2 (a known target of TGF $\beta$ ) did not appear to vary. Target genes of BMP signaling include inhibitor of differentiation 1 (Id1), $I d 2$ and $I d 3$ and Bmp2k (BMP2-inducible kinase) $)^{15,16}$. The microarray expression profiles of $I d 1, I d 3$ and $B m p 2 k$ were lower in the day phase and higher in the night phase (Figure 3D). Thus, the data support the hypothesis that BMP signaling in tendon is "gated" by the circadian clock, with an opposing pattern to the rhythmic expression of Grem2.

Gremlin-2 inhibits phosphorylation of Smad1/5 and calcium deposition. To determine if Gremlin-2 is capable of inhibiting BMP signaling we used an in vitro osteogenic differentiation model for human tenocytes. Addition of rhBMP2 to primary human tendon 
A

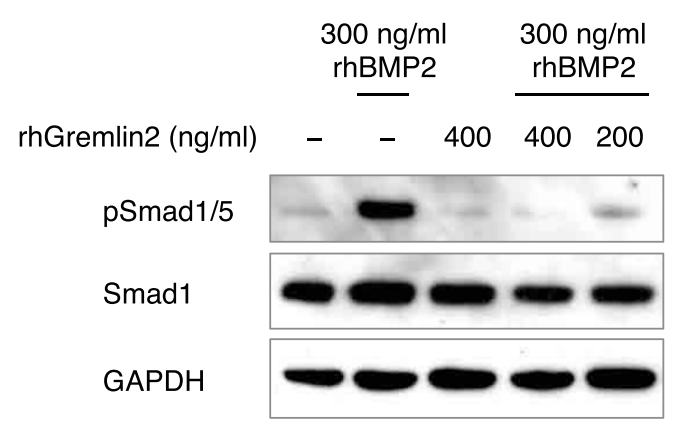

B
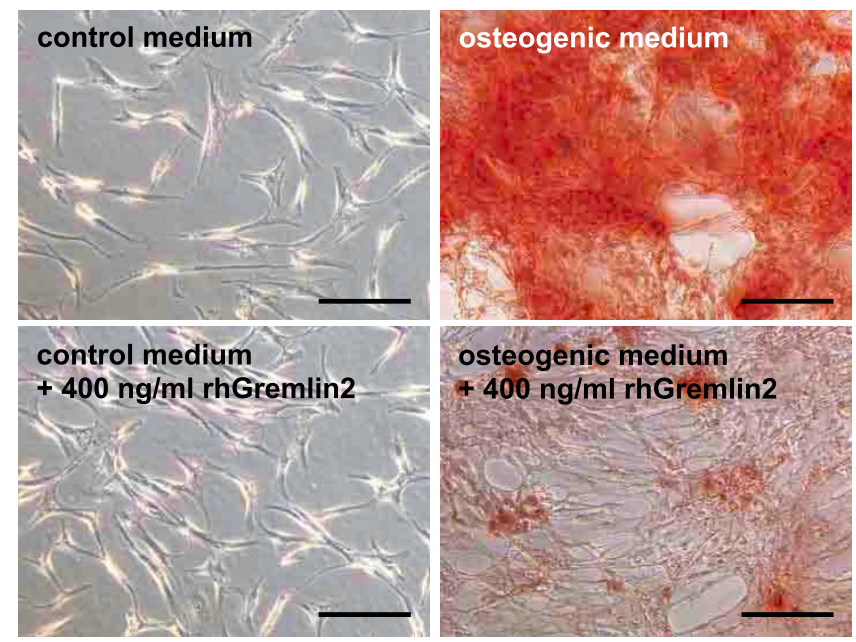

Figure $4 \mid$ Gremlin-2 inhibits BMP signaling and calcium deposition by human tendon cells. (A) Primary human tendon cells were treated with recombinant human BMP2 in the presence or absence of recombinant human Gremlin-2. Phosphorylation of Smad1/5 was examined by western blotting $(n=3)$. Western blot analysis of GAPDH protein served as a loading control. Full-length western blots are shown in Supplementary Figure S5. (B) Primary human tendon cells cultured in osteogenic medium in the presence or absence of $400 \mathrm{ng} / \mathrm{ml}$ recombinant human Gremlin-2 for 14 days were stained with Alizarin red $(n=3)$. Bars $=200 \mu \mathrm{m}$.

cells activated the phosphorylation of Smad1/5, which was reduced by pre-incubation with rhGremlin-2 (Figure 4A). Next, human tendon cells cultured in an osteogenic medium for 14 days stained bright red by Alizarin red, indicative of calcium deposition (Figure 4B). However, only low-level calcium deposition was observed in cells co-incubated with rhGremlin-2. Together, these data show that Gremlin-2 inhibits BMP signaling and calcium deposition in human tenocytes.

Aberrant BMP signaling and ectopic calcification in Achilles tendon of CLOCKA19 mice. The circadian control of Grem 2 and BMP activity prompted us to hypothesize that the CLOCK $\Delta 19$ mouse will have aberrant BMP signaling, predisposing the tendons to calcification. To test this, we first compared the expression of Grem 2 in CLOCK $\Delta 19$ with wild type tail tendons at two time points corresponding to the peak and trough expression of Grem2. Consistent with the loss of circadian rhythms in CLOCK $\Delta 19$ tendons (Figure 1C), we observed a lack of time-dependent expression for Grem2 (Figure 5A). Importantly, at the peak expression time of CT7 in wild type tendons, Grem 2 expression in CLOCK $\Delta 19$ tendon was significantly low. The low level of Grem 2 expression was associated with an aberrant pattern of phosphorylated Smad1/5 (Figure 5B). Noteworthy, low level BMP signaling seen in wild-type tendon at CT7 was replaced by elevated BMP signaling in the CLOCK $\Delta 19$
A

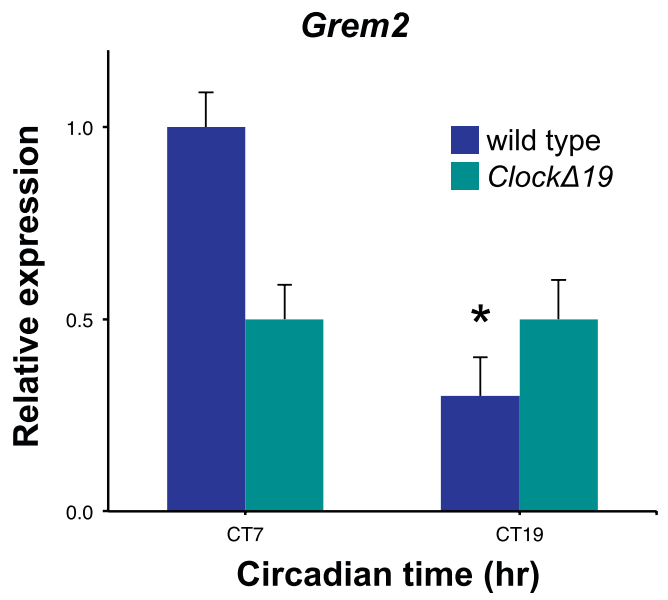

B

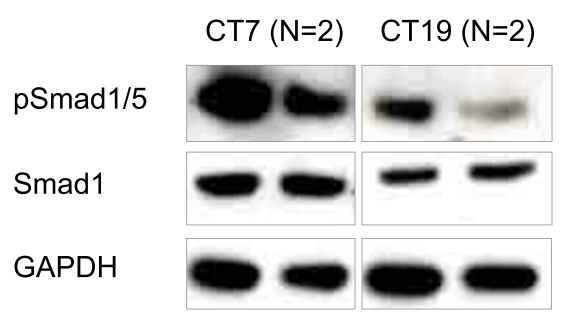

C

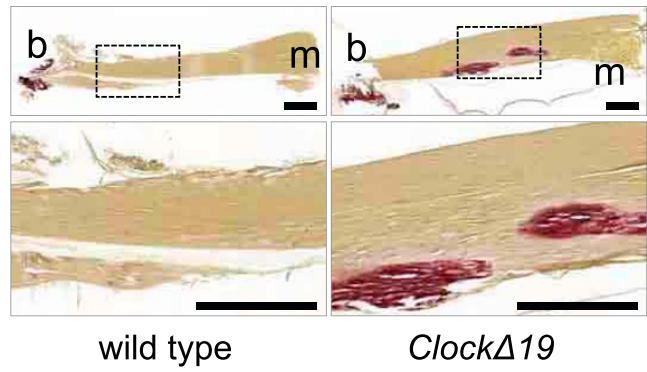

Figure $5 \mid$ Tendons from CLOCK $\Delta 19$ mice exhibit ectopic calcification. (A) Expression of Grem2 in tail tendons of CLOCKA19 mice compared with wild type tendons at CT7 (peak expression) and CT19 (nadir expression) $(\mathrm{n}=3)$. qPCR values normalized to GAPDH. $* p<0.05$, oneway ANOVA. Error bars indicate the SEM. (B) Protein was purified from CLOCK 19 tail tendons at CT7 and CT19 and phosphorylation of Smad1/ 5 were analyzed by western blotting $(n=2)$. Western blot analysis of GAPDH protein served as a loading control. (C) Longitudinal sections of Achilles tendon from 18 week-old wild type and CLOCKA19 mice stained with Alizarin red. $\mathrm{b}=$ attachment of tendon to bone, $\mathrm{m}=$ attachment of tendon to muscle. Bars $=500 \mu \mathrm{m}$.

tendons, suggesting an inhibitor function of the clock on BMP signaling. Alizarin Red staining of Achilles tendon showed the presence of calcification in tendons of CLOCK $\Delta 19$ mice but not in young age-matched wild type mice (Figure $5 \mathrm{C}$ ).

Aberrant clock in aged tendons is associated with ectopic calcification. To test if the tendon clock changes with ageing, we compared PER2::Luc rhythms in tendon from old (22-24 month old) and young adult (2-3 month old) mice (Fig 6A). There was a significant $40 \%$ reduction in the oscillation amplitude of PER2::Luc rhythms in aged tendon and the circadian phase was delayed by $\sim 6 \mathrm{hr}$ (Figure 6B-D). Comparison of the mRNA levels of core clock genes in young and old tendon showed significant dampening in the time-of-day dependent expression of Bmall and Per 2 and reduced levels of Nr1d1 (Figure 6E). Importantly, in parallel to the clock gene changes, the rhythmic expression of 

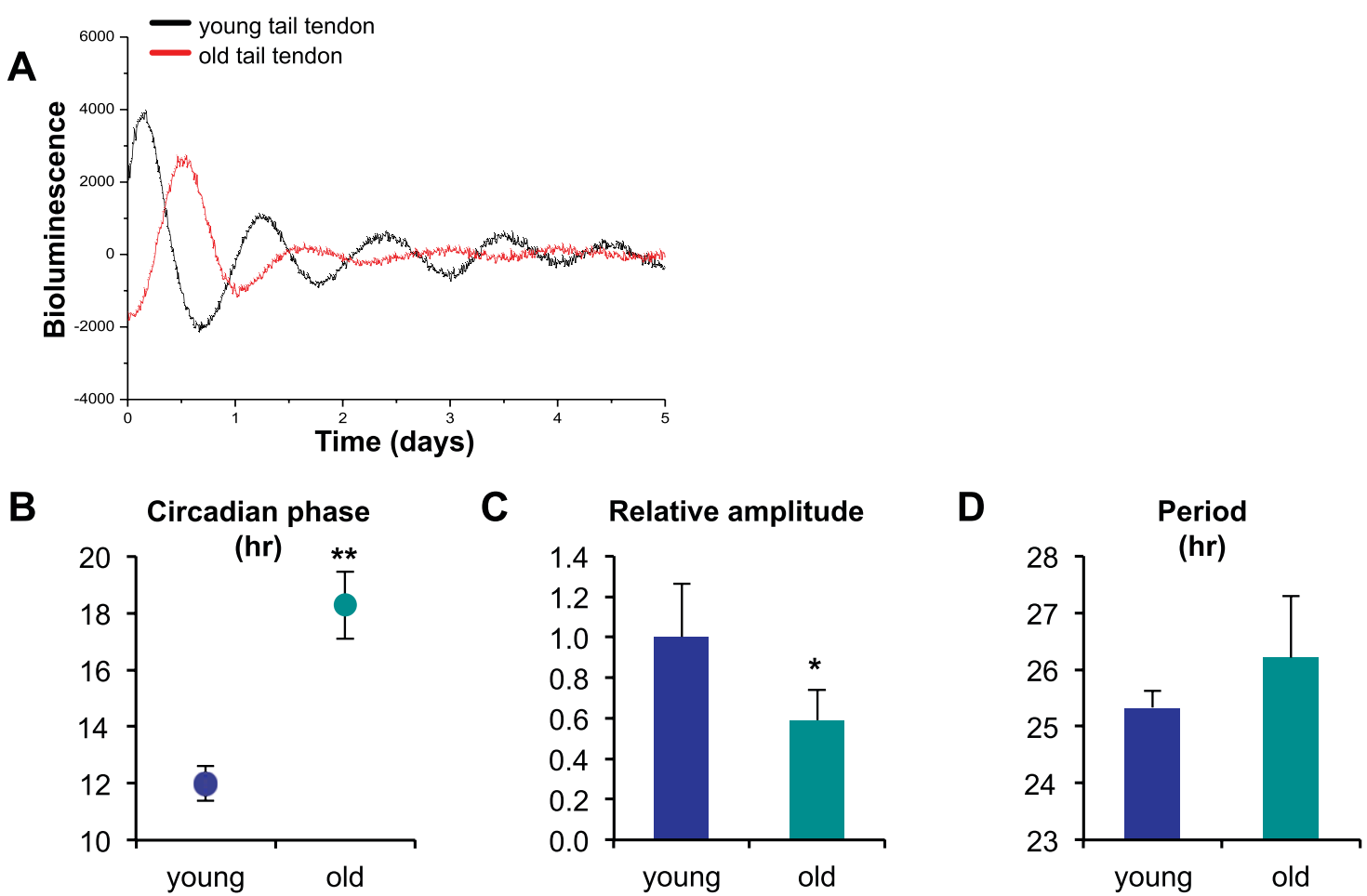

$\mathbf{E}$

Bmal1

Per2

Nr1d1
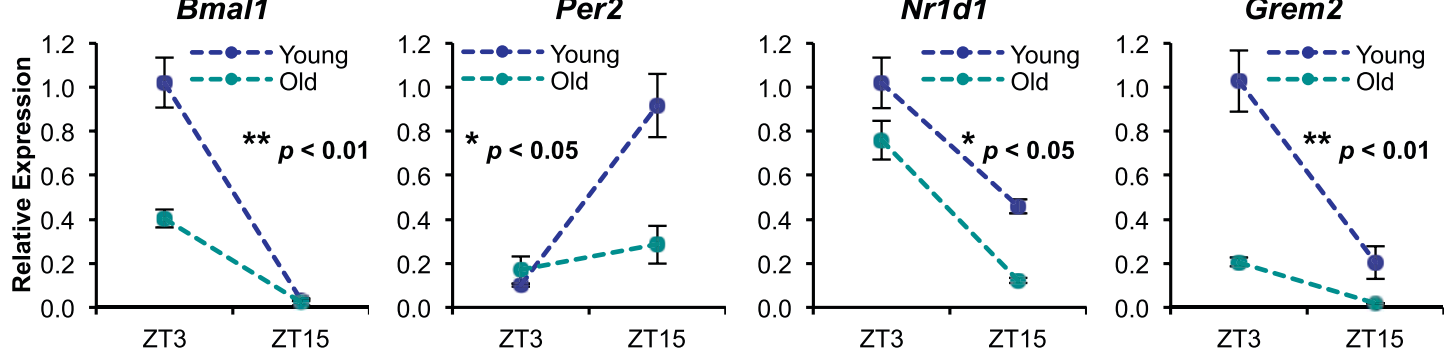

$\mathbf{F}$

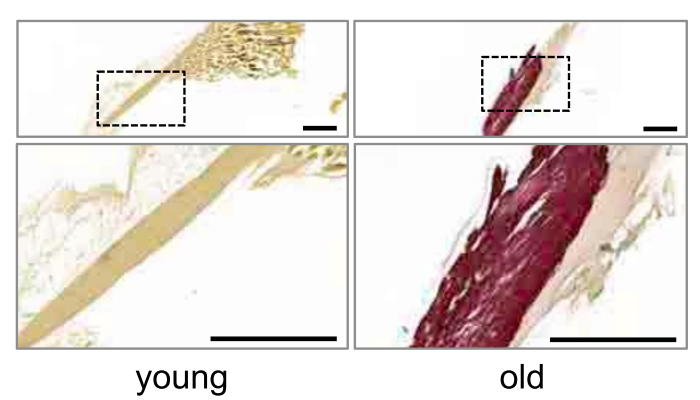

Figure 6 | Ectopic calcification in aged tendons correlates with disrupted circadian rhythm and altered Grem2 expression. (A) Representative PMT recordings (raw and normalized) of ex vivo tail tendons from young (2-3 months) and old (22-24 months) PER2::Luc mice ( $\mathrm{n}=3$ ). Comparison of (B) circadian phase, (C) amplitude and (D) period between young and aged groups $(\mathrm{n}=4)$. (E) Expression of clock genes, Bmal1, Per2 and Nr1d1 $(\mathrm{REV}-\mathrm{ERB} \alpha)$, and Grem 2 in tail tendon of young (2-3 months) and old (22-24 months) at ZT3 and ZT15 ( $\mathrm{n}=2$ ). qPCR values normalized to GAPDH. ${ }^{*} \mathrm{p}<0.05,{ }^{* *} \mathrm{p}<0.01$, unpaired t-tests. Error bars indicate the SEM. (F) Alizarin red staining of longitudinal sections of Achilles tendon from young (2-3 months) and aged (22-24 months) wild type mice. Bars $=500 \mu \mathrm{m}$.

Grem2 seen in young tendon was severely dampened in the old tendon. Finally, the aged wild-type mice (22-24 month old) developed calcific deposits in their Achilles tendons (Figure 6F).

\section{Discussion}

We have identified cell-autonomous oscillations of clock genes in murine and human tenocytes and shown that the circadian clock is a master regulator of BMP signaling. These results provide a basis for exploring the links between tendon clocks, ageing and tendinopathies.
Circadian clock disruptions have been linked to various pathologies and diseases, including defects in the musculoskeletal system. For instance, bone volume is significantly increased in the Per2 mutant and Cry 2 knockout mice, caused by defects in osteoblasts and osteoclasts, respectively ${ }^{17}$. Furthermore, shorter bone length was reported in the Bmal1 knockout mouse because of defects in chondrocyte differentiation in the growth plate ${ }^{18}$. Moreover, maximal muscle force was reduced (by 30\%) in the Bmal1-null and CLOCK mutant mice ${ }^{19}$. Consistently, by comparing the tendon circadian transcriptome to that of the cartilage ${ }^{9}$ and muscle ${ }^{8}$, we found that 
these tissues had few rhythmic genes in common. In fact, the majority of the common rhythmic genes encoded components of circadian clocks.

Interestingly, the initial phenotypic characterization of the Mop3 (Bmal1) null mouse reported ectopic calcification in tendons and ligaments, among other signs of non-inflammatory arthropathy ${ }^{10}$. The underlying mechanisms for the calcified tendonopathies have never been addressed. Our results showing calcification in the CLOCK $\Delta 19$ mouse tendons, support a hypothesis that the CLOCK/ BMAL1 complex regulates key signaling pathways that inhibit ectopic calcification in normal rhythmic mice. Thus, when the circadian rhythm is disrupted, the tendons are predisposed to calcification.

BMPs are the largest class of signaling ligands in the TGF $\beta$ superfamily and regulate a wide variety of processes during embryo patterning, skeletal and non-skeletal development, differentiation of cell fate, and have additional roles in cancer and tumor progression $\left(\right.$ reviewed $b y^{20}$ ). The basic signaling mechanism is the same for all TGF $\beta$ superfamily members and is initiated when ligand dimers engage with type I and type II serine/threonine kinase receptors. In the activated receptor complex, the type II receptor phosphorylates the type I receptor which provides a binding site for the receptorregulated SMADs (R-SMADs). The plethora of normal biological processes triggered by BMP signaling, and the involvement of the pathway in pathological conditions including cancer, is partly explained by the large number of BMP ligands ( $~ 20$ in number), the choice of type I and type II receptors (12 in total) for ligand engagement, and the availability of SMAD dependent and SMADindependent signal transduction. With such multiple opportunities for pathway activation, the blockade of BMP ligand-receptor engagement by extracellular antagonists is a crucial on-off switch of the signaling pathway. Thus, the circadian clock could be a super-regulator of BMP signaling by regulating the expression of Grem2.

Gremlins are small, single domain proteins that contain a cysteine-rich fold that occurs in members of the DAN/Cerberus family of secreted BMP antagonists ${ }^{21-25}$. Gremlin-1 prevents binding of BMP receptor binding but does not affect the activity of other members of the TGF $\beta$ superfamily ${ }^{13}$. Gremlin-2 (also known as Protein Related to Dan and Cerberus, PRDC) is the second member of the Gremlin family and can inhibit ligand signaling by BMP 2 and BMP4 but has minimal effects on GDF-9, activin and TGF $\beta$ signaling $^{26}$. Previous studies have shown that Grem2 restricts BMP signaling to the ventral arches and thereby promotes dorsal and intermediate skeletal fates ${ }^{27}$, is necessary for cardiac laterality and atrial differentiation ${ }^{28}$, and can promote the differentiation of stem cells to cardiomyocytes ${ }^{29}$. However, a role for Grem 2 in tendon homeostasis and calcification had not been previously described. BMP signaling has been shown to drive osteogenic differentiation of tendon-derived stem cells ${ }^{30}$. Furthermore, BMP2 and BMP7 can be detected in the subacromial bursa of patients with chronic rotator cuff degeneration at levels sufficient to induce osteogenic differentiation of mouse myoblast-derived $\mathrm{C} 2 \mathrm{C} 12 \mathrm{cells}^{31}$.

Analysis of our microarray data showed that Grem2 (but not Bmp2, Bmp4 and Bmp7) was rhythmically expressed in tendon. The rhythmic expression of Grem2 was validated in mouse Achilles and tail tendon and primary human tendon cells. In further experiments we showed that at the peak expression time of CT7, Grem 2 expression in CLOCK $\Delta 19$ mutant tail and Achilles tendons was significantly lower than in wild-type tendons, thus indicating a role of the molecular circadian clock in regulating Grem2 expression in tendon. We also showed that BMP-signaling target genes (Id1, Id3 and $B m p 2 k$ ) are rhythmic, indicating circadian activity of the BMP signaling. A mechanistic link between Gremlin-2 and tendon calcification was evident when Gremlin-2 was capable of inhibiting BMP-induced calcification of human tenocytes in culture, revealing Gremlin-2 as a novel potent antagonist of BMP signaling in tendon.
Consistent with the inhibitory role of Gremlin-2, we observed a profound day/night difference in the Smad1/5 phosphorylation opposing the levels of Grem2 expression. Therefore, the circadian control of Grem2 is likely to be involved in "gating" the BMP signaling pathway in a rhythmic manner.

It is well established that systemic circadian rhythms dampen with aging as shown by body temperature cycles and hormones ( $\mathrm{se}^{32}$ and references therein). Likewise, we showed that tendon rhythms with altered phase and reduced oscillation amplitude are a feature of aging mice. The delayed phase implies that there is an internal desynchrony between tendon clocks and the central brain clock, which might disrupt the temporal coordination of the tendon rhythmic genes. Such desynchrony in gene expression is exacerbated by the reduced amplitude, leading to compromised tissue homeostasis and increased risk of pathology. As age is a risk factor for calcific tendinopathies in elderly humans, we envisage a scenario in which chronic circadian misalignment/disruption (e.g. during aging) predisposes tendon tissue to calcific changes. Future studies in humans and the use of conditional mutant mouse models may reveal more information about the tendon clock as an important regulator of tissue physiology and pathology.

\section{Methods}

Animals. All animal work was approved by The University of Manchester Breeding and Supply Unit and was in accordance with the 1986 Home Office Animal Procedures Act (UK), and following local ethical review.

Tendon Explant Cultures and Bioluminescence Recording. Tendon cultures from PER2::Luc mice were prepared by dissecting out the Achilles and tail tendons. Tendon explants were cultured on $0.4 \mu \mathrm{m}$ cell culture inserts (Millipore) and bioluminescence was recorded in real-time using as previously described ${ }^{33}$. Dexamethasone (100 nM) was added to the recording media to re-initiate circadian rhythms. Baseline subtraction, period, phase and amplitude calculation was carried out as previously described $^{9}$. Cultures were also visualized using a self-contained Luminoview LV200 microscope (Olympus) and recorded using a cooled Hamamatsu ImageEM C9100-13 EM-CCD camera. Images were taken every hour for 4 days, and combined in ImageJ.

Time Series Microarrays and Quantitative PCR. The circadian transcriptome studies in tendon were performed as described before ${ }^{9}$. Tail tendons were collected every $4 \mathrm{hr}$ commencing at circadian time CT3 ( $3 \mathrm{hr}$ into the light phase), for $48 \mathrm{hr}$. Tissues ( 6 samples per time point) were snap frozen in liquid nitrogen. Two samples per time point were used for microarray; the remainder was used for qPCR validation. RNA extractions were carried out using TRIzol reagent (Invitrogen) according to manufacturer's protocol. Mouse430_2 Affymetrix GeneChips were run according to manufacturers' instructions. Raw data were deposited in Array Express (accession number pending). CircWave v5 (by Dr. Roelof Hut, cut-off point of $\mathrm{q}<0.003$ ) and JTK-Cycle ${ }^{34}$ (q-value of 0.1 ) were used to identify circadian transcripts as described previously' . Local false discovery rate was calculated using an R package 'fdrtool' ${ }^{\prime 35}$. To be stringent, genes identified using both methods are counted as positive. To model the data for clustering, we used a Gaussian process model ${ }^{9}$. Validation of time-series arrays and gene expression analyses was examined by qPCR (for primers see Supplementary Table 1) as described previously ${ }^{36}$.

Primary Mouse and Human Tenocytes Culture. Cells from tail tendons were released by treatment with $1000 \mathrm{U} / \mathrm{ml}$ bacterial collagenase type 4 (Lorne Laboratories) in $0.25 \%$ (wt/vol) trypsin (Invitrogen). Cells were grown at $37 \mathrm{oC}$ until 90-100\% confluent prior to synchronization and PMT recording. Primary human tenocytes were obtained as described previously ${ }^{37}$. Cells from three preparations were used in the experiments described in this study. For time course experiments, confluent cells were synchronized with DMEM:F12 containing 50\% (v/v) horse serum (Invitrogen), L-G and P/S for $2 \mathrm{hr}$ at $37 \mathrm{oC}$. RNA was isolated after $24 \mathrm{hr}$, at 4 $\mathrm{hr}$ intervals for $20 \mathrm{hr}$. For treatment with recombinant human BMP2 (PHC7145, Invitrogen) and recombinant human Gremlin-2 (SRP4657, Sigma), 90-100\% confluent cells were washed with PBS and incubated with serum-free media for $1 \mathrm{hr}$ at $37 \mathrm{oC}$. Then, cells were treated with BMP2 that had been pre-incubated for $1 \mathrm{hr}$ at $4 \mathrm{oC}$ with Gremlin-2 in serum-free medium. Protein was isolated after $24 \mathrm{hr}$ and analyzed by western blotting.

Lentiviral Transduction. Lentiviral transduction of primary human tenocytes was performed using methods previously described ${ }^{9}$.

Protein Extraction and Western Blotting. Proteins from tail tendons were extracted

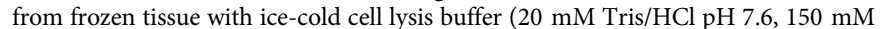
$\mathrm{NaCl}, 1 \mathrm{mM}$ ethylene diamine tetra-acetic acid (EDTA), 1\% (v/v) Igepal CA-630, $50 \mathrm{mM}$ sodium fluoride) containing complete, mini EDTA-free protease inhibitor cocktail and PhosSTOP phosphatase inhibitor cocktail (Roche). Lysates were cleared 
by centrifugation at $10000 \times \mathrm{g}$, at $4 \mathrm{oC}$. Commercially available antibodies used to detect phospho-Smad1/5 (\#9516), Smad1 (\#6933), Smad5 (\#9517), phospho-Smad2 (\#3108) and Smad2 (\#3103) were purchased from Cell Signaling Technologies. GAPDH was detected using a monoclonal antibody from Sigma (G8795).

Calcium Deposition Assay and Alizarin Red Staining. Achilles tendons were fixed in 2\% (w/v) PFA in PBS for 20 minutes, wax processed. 5-6 $\mu \mathrm{m}$ sections were dewaxed before staining. Calcium deposition in primary human tenocytes was induced by culturing cells in Osteogenic Medium (Lonza) according to the manufacturer's protocol. After 14 days, cells were fixed with $2 \%$ (w/v) PFA in PBS for 20 minutes. Fixed cells or tissue sections were washed with distilled water and stained with $2 \%(\mathrm{w} /$ v) Alizarin Red S, pH 4.2 for $10 \mathrm{~min}$. Washed plates or sections were air-dried and imaged using a Pannoramic slide scanner and Pannoramic Viewer software (3DHISTECH).

Statistical Analyses. Mean and standard error of the mean were calculated for each data set from at least three biological replicates (indicated by the $\mathrm{n}$ in figures). Data was evaluated using two sample t-tests with GraphPad Prism (GraphPad Software). Differences were considered significant at the values of $\mathrm{p}<0.05(*), \mathrm{p}<0.01\left({ }^{* *}\right)$ or $\mathrm{p}$ $<0.0001(* * *)$.

1. Chaudhury, S., Holland, C., Thompson, M. S., Vollrath, F. \& Carr, A. J. Tensile and shear mechanical properties of rotator cuff repair patches. J Shoulder Elbow Surg 21, 1168 (2012).

2. Butler, D. L. et al. Functional tissue engineering for tendon repair: A multidisciplinary strategy using mesenchymal stem cells, bioscaffolds, and mechanical stimulation. J Orthop Res 26, 1 (2008).

3. Oliva, F., Barisani, D., Grasso, A. \& Maffulli, N. Gene expression analysis in calcific tendinopathy of the rotator cuff. Eur Cell Mater 21, 548 (2011).

4. Lippmann, R. K. Observations concerning the calcific cuff deposit. Clin Orthop 20, 49 (1961).

5. Welfling, J., Kahn, M. F., Desroy, M., Paolaggi, J. B. \& de Seze, S. [Calcifications of the shoulder. II. The disease of multiple tendinous calcifications]. Rev Rhum Mal Osteoartic 32, 325 (1965)

6. Uhthoff, H. K. \& Loehr, J. W. Calcific Tendinopathy of the Rotator Cuff: Pathogenesis, Diagnosis, and Management. J Am Acad Orthop Surg 5, 183 (1997).

7. Oliva, F., Via, A. G. \& Maffulli, N. Physiopathology of intratendinous calcific deposition. BMC Med 10, 95 (2012).

8. McCarthy, J. J. et al. Identification of the circadian transcriptome in adult mouse skeletal muscle. Physiol. Genomics 31, 86 (2007).

9. Gossan, N. et al. The circadian clock in murine chondrocytes regulates genes controlling key aspects of cartilage homeostasis. Arthritis Rheum 65, 2334 (2013).

10. Bunger, M. K. et al. Progressive arthropathy in mice with a targeted disruption of the Mop3/Bmal-1 locus. Genesis 41, 122 (2005).

11. Vitaterna, M. H. et al. Mutagenesis and mapping of a mouse gene, Clock, essential for circadian behavior. Science 264, 719 (1994).

12. Yoo, S. H. et al. PERIOD2::LUCIFERASE real-time reporting of circadian dynamics reveals persistent circadian oscillations in mouse peripheral tissues. Proc Natl Acad Sci U S A 101, 5339 (2004).

13. Hsu, D. R., Economides, A. N., Wang, X., Eimon, P. M. \& Harland, R. M. The Xenopus dorsalizing factor Gremlin identifies a novel family of secreted proteins that antagonize BMP activities. Mol Cell 1, 673 (1998).

14. Miyazawa, K., Shinozaki, M., Hara, T., Furuya, T. \& Miyazono, K. Two major Smad pathways in TGF-beta superfamily signalling. Genes Cells 7, 1191 (2002)

15. Hollnagel, A., Oehlmann, V., Heymer, J., Ruther, U. \& Nordheim, A. Id genes are direct targets of bone morphogenetic protein induction in embryonic stem cells. I Biol Chem 274, 19838 (1999).

16. Yang, J. et al. Id proteins are critical downstream effectors of BMP signaling in human pulmonary arterial smooth muscle cells. Amer I Physiol (2013).

17. Maronde, E. et al. The clock genes Period 2 and Cryptochrome 2 differentially balance bone formation. PloS one 5, e11527 (2010).

18. Takarada, T. et al. Clock genes influence gene expression in growth plate and endochondral ossification in mice. J Biol Chem 287, 36081 (2012).

19. Andrews, J. L. et al. CLOCK and BMAL1 regulate MyoD and are necessary for maintenance of skeletal muscle phenotype and function. Proc Natl Acad Sci U S A 107, 19090 (2010)

20. Wakefield, L. M. \& Hill, C. S. Beyond TGFbeta: roles of other TGFbeta superfamily members in cancer. Nat Rev Cancer 13, 328 (2013).

21. Topol, L. Z. et al. Biosynthesis, post-translation modification, and functional characterization of Drm/Gremlin. J Biol Chem 275, 8785 (2000).

22. Topol, L. Z. et al. Identification of drm, a novel gene whose expression is suppressed in transformed cells and which can inhibit growth of normal but not transformed cells in culture. Mol Cell Biol 17, 4801 (1997).

23. Bragdon, B. et al. Bone morphogenetic proteins: a critical review. Cell Signal 23, 609 (2011).

24. McMahon, R. et al. IHG-2, a mesangial cell gene induced by high glucose, is human gremlin. Regulation by extracellular glucose concentration, cyclic mechanical strain, and transforming growth factor-beta1. J Biol Chem 275, 9901 (2000).

25. Wordinger, R. J., Zode, G. \& Clark, A. F. Focus on molecules: gremlin. Exp Cell Res 87, 78 (2008).

26. Sudo, S., Avsian-Kretchmer, O., Wang, L. S. \& Hsueh, A. J. Protein related to DAN and cerberus is a bone morphogenetic protein antagonist that participates in ovarian paracrine regulation. J Biol Chem 279, 23134 (2004).

27. Zuniga, E., Rippen, M., Alexander, C., Schilling, T. F. \& Crump, J. G. Gremlin 2 regulates distinct roles of BMP and Endothelin 1 signaling in dorsoventral patterning of the facial skeleton. Development 138, 5147 (2011).

28. Muller, II et al. Functional modeling in zebrafish demonstrates that the atrialfibrillation-associated gene GREM2 regulates cardiac laterality, cardiomyocyte differentiation and atrial rhythm. Dis Model Mech 6, 332 (2013).

29. Tanwar, V. et al. Gremlin 2 promotes differentiation of embryonic stem cells to atrial fate by activation of the JNK signaling pathway. Stem Cells (2014).

30. Rui, Y. F. et al. Mechanical loading increased BMP-2 expression which promoted osteogenic differentiation of tendon-derived stem cells. J Orthop Res 29, 390 (2011).

31. Neuwirth, J. et al. Expression of bioactive bone morphogenetic proteins in the subacromial bursa of patients with chronic degeneration of the rotator cuff. Arthritis Res Therapy 8, R92 (2006).

32. Brown, S. A., Pagani, L., Cajochen, C. \& Eckert, A. Systemic and cellular reflections on ageing and the circadian oscillator: a mini-review. Gerontology 57, 427 (2011).

33. Meng, Q. J. et al. Ligand modulation of REV-ERBalpha function resets the peripheral circadian clock in a phasic manner. J Cell Sci 121, 3629 (2008).

34. Hughes, M. E., Hogenesch, J. B. \& Kornacker, K. JTK_CYCLE: an efficient nonparametric algorithm for detecting rhythmic components in genome-scale data sets. J Biol Rhythms 25, 372 (2010).

35. Strimmer, K. fdrtool: a versatile R package for estimating local and tail area-based false discovery rates. Bioinformatics 24, 1461 (2008).

36. Livak, K. J. \& Schmittgen, T. D. Analysis of relative gene expression data using real-time quantitative PCR and the 2(-Delta Delta C(T)) Method. Methods 25, 402 (2001).

37. Bayer, M. L. et al. The initiation of embryonic-like collagen fibrillogenesis by adult human tendon fibroblasts when cultured under tension. Biomaterials 31, 4889 (2010).

\section{Acknowledgments}

We thank David F. Holmes for his help with this project. Ding Jin for help with genotyping and mouse colony maintenance. We thank Leo Zeef and Magnus Rattray for their help with the microarray data analysis. We thank the Electron Microscopy Facility, Genomic Technologies and Bioimaging in the Faculty of Life Sciences (University of Manchester) for providing technical support/advice. We also thank Dr. Michael Hughes (Yale School of Medicine) for providing the JTKCycle script, Dr. Roelof Hut (University of Groningen) for Circwave software, and Prof. Joseph Takahashi (University of Texas Southwestern Medical Center) for PER2::Luc and Clock $\Delta 19$ mice. This work was supported by a Career Development Award (G0900414) to QJM from the Medical Research Council, UK, a 5-year Programme Grant (091840/Z/10/Z) to KEK from the Wellcome Trust, a Core funding award (Wellcome Trust $088785 / Z / 09 / Z$ ) to the University of Manchester Wellcome Trust Centre for Cell-Matrix Research, and the BBSRC for funding the purchase of the Olympus Luminoview LV200 microscope (Olympus) and Hamamatsu ImageEM C9100-13 EM-CCD camera (BB/J003441). C.-Y.C.Y. was funded partly by a PhD studentship from the BBSRC and partly by a grant from the University of Manchester.

\section{Author contributions}

The author(s) have made the following declarations about their contributions: Conceived the project: K.E.K. Conceived and designed the experiments: K.E.K., Q.-J.M., C.-Y.C.Y. Performed the experiments: C.-Y.C.Y., N.G., Q.-J.M., Y.L., A.H. Contributed reagents/ materials/analysis tools: J.J.H., Q.-J.M., M.L.B., M.K. Wrote the paper: K.E.K., Q.-J.M.

\section{Additional information}

Supplementary information accompanies this paper at http://www.nature.com/ scientificreports

Competing financial interests: The authors declare no competing financial interests. How to cite this article: Yeung, C.-Y.C. et al. Gremlin-2 is a BMP antagonist that is regulated by the circadian clock. Sci. Rep. 4, 5183; DOI:10.1038/srep05183 (2014).

his work is licensed under a Creative Commons Attribution 3.0 Unported License. The images in this article are included in the article's Creative Commons license, unless indicated otherwise in the image credit; if the image is not included under the Creative Commons license, users will need to obtain permission from the license holder in order to reproduce the image. To view a copy of this license, visit http://creativecommons.org/licenses/by/3.0/ 